\title{
Problematiche insediative nel distretto di Limassol (Cipro) tra tarda età del bronzo e prima età del ferro
}

\author{
Serena Torello Di Nino
}

Lo sviluppo dei modelli insediativi nel distretto di Limassol si adatta alle caratteristiche geomorfologiche e alla distribuzione delle risorse minerarie di tale regione che costituiscono l'elemento fondamentale di lettura, tanto da determinare la ratio del quadro insediativo (fig.1).

La conformazione geologica dell'area consta sia di rocce di origine vulcanica, fortemente metallifere, sia di formazioni sedimentarie, dal punto di vista morfologico fortemente caratterizzate da sistemi vallivi che scendono dalle pendici dei Troodos, scorrendo, con andamento nord-sud, perpendicolarmente alla costa. I principali bacini idrografici come quelli del fiume Kouris a ovest, i fiumi Garyllis e Germasogeia al centro e il complesso bacino idrografico pluriramificato del Pyrgos-Moni a est, determinano bacini geomorfologici ed idrografici autonomi. I modelli insediativi si adattano fortemente al contesto topografico e l'elemento di forte caratterizzazione e differenziazione è costituito da presenza e ricchezza delle vene metallifere che ovviamente cambiano da valle a valle, creando una microzonizzazione molto interessante, sì da determinare un fenomeno di micro-regionalismi.

La regione meridionale di Cipro, e in particolare il distretto di Limassol, ha evidenziato per il Tardo Bronzo modelli insediativi basati su un policentrismo funzionale e gerarchizzato molto caratteristico, piuttosto che un modello gerarchico a singolo sito egemone, come accade per il distretto regionale orientale dell'isola, o anche a confronto con quanto accade nella stessa area d'indagine sino al Bronzo Medio. Il numero dei siti è triplicato rispetto al $\mathrm{MC}$, aumento verificatosi anche altrove, ma non nella stessa misura, come nel sud di Cipro. Inoltre, la variazione del numero dei siti a sud nell'intera età del Bronzo (antico, medio, tardo) differisce da quella che si verificò nel resto dell'isola: questa rappresenta senz'altro una caratteristica importante che aiuta a definire questo contesto regionale. Sembrerebbe infatti che la regione meridionale di Cipro sia caratterizzata da una percentuale molto elevata dei siti più ricchi dell'isola durante il $\mathrm{TC}^{1}$ (cioè la tarda età del Bronzo a Cipro); tale ricchezza e importanza non possono essere solo il risultato di attività legate all'agricoltura: esse dovettero infatti avere funzioni differenziate e complementari nello sfruttamento delle risorse agricole quanto minerarie. Inoltre, i siti costieri dovettero svolgere un ruolo fondamentale lungo rotte di commercio e di cabotaggio grazie alla loro portualità naturale, svolgendo una funzione commerciale non solo interna, ma anche relativamente a contatti con il Mar Egeo ed il Vicino Oriente. D'altro canto tali porti hanno probabilmente svolto un ruolo importante nella comunicazione via mare tra i siti della regione e gli altri insediamenti di Cipro, probabilmente perché le montagne meridionali e settentrionali ne impedivano tale comunicazione. Bisogna poi pensare che con il tempo vi sia stata una differenziazione nella gerarchia degli approdi, tanto che alcuni divennero porti strutturati, mentre altri rimasero approdi naturali dalla frequentazione meno ampia. Un esempio calzante di approdo che andò strutturandosi con il tempo, proprio nella prima età del Ferro, è il noto porto di Amathus, in parte sotto il livello del mare e in parte interrato già in età tardo-imperiale. Invece un sito inedito che potrebbe essere esemplificativo del caso di

\footnotetext{
${ }^{1}$ Catling 1962, pp. 110-112.
} 
approdi naturali utilizzati tra tardo Bronzo e primo Ferro, ma che non vennero mai dotati di infrastrutture complesse, potrebbe essere quello di Agios Georghios Alamanos $^{2}$, posto a sud dell'isola di Cipro (a circa $8 / 10 \mathrm{~km}$ a est di Limassol), che sembra avere tutte le caratteristiche di un insediamento nato per il cabotaggio costiero. Le coste si prestano come punto d'approdo per un cabotaggio a breve distanza. Oltre al rinvenimento di due capanne scavate sulla falesia, di cui una sulla falesia, mentre l'altra, per un crollo, oggi è sita sotto il livello del mare, sembrerebbe esserci un'altra prova: ad est di tali emergenze, è stato individuato una bitta d'attracco che proverebbe tale ruolo ${ }^{3}$.

Spostandoci ad est del distretto di Limassol, una serie di insediamenti sono distribuiti su tale modello "insediamento-porto" come quello nel moderno villaggio di Pyla, Kition nella moderna Larnaca ed Hala Sultan Tekke nei pressi del lago salato di Larnaka, costituito da un ingresso aperto ${ }^{4}$. Kition, più ad ovest rispetto a questi insediamenti, nella zona intorno a Maroni sulla riva occidentale del fiume Pendaskinos ha attirato gran parte del commercio con l'occidente grazie al ritrovamento di un numero relativamente elevato di importazioni dall'Egeo.

Poi esistevano un'altra tipologia di insediamenti importanti caratterizzati da una localizzazione che si attesta sempre nella media valle di ogni bacino idrografico, non solo e banalmente perché interagivano con i fiumi come riserva idrica, ma soprattutto per il contesto geo-morfologico che tali territori presentavano sempre nella media valle, con ampi terrazzi fluviali, fertili e pianeggianti, utilissimi per lo sfruttamento agricolo. Tanto è vero che spesso tali insediamenti erano posti in aree che controllavano direttamente i campi circostanti e recavano evidenti segni di produzione e stoccaggio, come ad esempio gli insediamenti Episkopi-Bamboula, Erimi-Pitharka lungo il fiume Kouris ${ }^{5}$. Tali siti inoltre erano estremamente funzionali lungo i percorsi viari naturali perché fungevano da stazioni intermedie tra aree minerarie e aree portuali. In tal senso si potrebbe ipotizzare che alcuni di questi siti possa avere avuto un qualche ruolo nella fase di lavorazione del metallo per renderlo prodotto finito prima dell'esportazione.

D'altronde un fattore primario che ha contribuito all'aumento del numero e della ricchezza degli insediamenti nella regione di Limassol, nonché alla gerarchizzazione e differenziazione funzionale dei siti in questo territorio, è sicuramente il metallo che ha promosso Cipro come maggiore produttrice ed esportatrice nel Mediterraneo durante l'età del Bronzo, il rame.

Un certo numero di insediamenti di TC erano posti nei pressi delle risorse di rame, tanto che l'economia della regione era basata sull'estrazione e lavorazione del metallo. Si tratta di quei siti che Bernard Knapp definisce "siti minerari", con una vocazione economica legata ai processi estrattivi e di prima lavorazione del metallo, come nel caso di Sanida, lungo la valle di Moni, che funge da centro importante in piena area mineraria, con una tale specializzazione tecnologica da divenire anche

\footnotetext{
${ }^{2}$ Sito individuato nel 2014 dalla missione della Prof.ssa Oliva Menozzi, Università G. d'Annunzio di Chieti-Pescara.

${ }^{3}$ MenOZZI ET AL. 2014.

${ }^{4}$ CATLING 1962, pp. 136,142.

${ }^{5} \mathrm{Al}$ di fuori di tale distretto un confronto si può trovare con il sistema insediativo intorno all'area di Paleopaphos.

${ }^{6}$ KNAPP 1986.
} 
centro di produzione della White Slip Ware, che presuppone un livello tecnologico avanzato ${ }^{7}$.

Tale differenziazione nella localizzazione e nella funzione dei siti determina una gerarchia nel loro utilizzo e un adattamento del modello insediativo al contesto geomorfologico che può anche variare da vallata a vallata per l'area di Limassol, ma si tratta comunque di una matrice insediativa comune con varianti locali, che attesta una sorta di micro-regionalismo sempre però facente capo ad una comune matrice.

Scendendo più nello specifico, il distretto di Limassol consta di 3 modelli insediativi in tre aree che in realtà potremmo vedere come varianti di un unico modello insediativo in contesti micro-regionali nell'ambito della macro-area del distretto di Limassol.

Una prima area è quella più ad ovest, è relativa alla valle del Kouris; una seconda area è quella dei sistemi vallivi paralleli di Polemyda e di Germasioa posti nell'area centrale; la terza area, più ad est è quella invece del sistema idrografico Pyrgos/Moni, che rappresenta un bacino idrografico molto più complesso e differenziato.

Le tre aree coincidono con progetti di studio del territorio, per cui tali distretti sono stati in qualche caso oggetto di ricognizioni intensive, in altri casi invece si è praticata un'archeologia d'emergenza con un dettaglio puntuale ma senza un'indagine intensiva sul territorio e sul contesto. Ciò determina una disomogeneità dei dati perché l'approccio e la strategia di campionatura nella ricerca e nell'interpretazione sono molto diversi, ad esempio le aree I e III (figg.2-3) sono state indagate intensivamente con progetti di ricerca $(\mathrm{KVP} \text { e } \mathrm{MPM})^{8}$ basati su una strategia di campionatura molto alta, invece per l'area II, area fortemente urbanizzata che è stata analizzata esclusivamente in base ai rinvenimenti da scavi da emergenza, spesso decontestualizzati, non è stato possibile ipotizzare una variante del modello insediativo, anche se è certo ravvisabile la matrice del modello insediamentale comune.

Dal punto di vista più generale quindi il modello insediativo del distretto di Limassol nella tarda età del bronzo e nelle prime fasi dell'età del ferrro, si presenta sostanzialmente basato su attracchi portuali regolari ma non tutti strutturati, che creano una base per smercio-commercio del metallo e approviggionamento di altre merci. Le aree della media valle di Moni, Pyrgos e Germasoia, così come nella Kouris presentano un policentrismo con una differenziazione di insediamenti che gerarchicamente fungono da centri di produzione e/o stoccaggio, ma anche utilizzati come centri di seconda lavorazione del rame, aree cultuali/santuariali lungo le vie che mettono in relazione tali villaggi che possiamo definire intermedi e le risorse minerarie. A monte di tali valli fluviali si trovano i distretti minerari, nell'area ove è sita Sanidha, alle sorgenti del Moni, tra Mazokambos e Kellaki, nel retroterra del Pyrgos, nell'area delle sorgenti del fiume Germasoia e nel retroterra dietro Polemida. Tali distretti minerari erano ovviamente sedi di centri estrattivi, ove avveniva anche la prima lavorazione e dove la tecnologia della lavorazione del rame, permetteva anche un avanzato livello nella tecnologia della ceramica, come ad esempio appunto Sanidha.

\footnotetext{
${ }^{7}$ TODD 1991, TODD-PILIDES 1992,2000

${ }^{8}$ Kouris VAlley Project (Università di Torino con la direzione del Dott. Luca Bombardieri) e MoNI Pyrgos Monagroulli Project (Università di Chieti con la direzione della Prof.ssa Oliva Menozzi).
} 
La valle del Kouris presenta un sistema d'insediamento piramidale in cui Alassa, per tutto il TC II, fungeva da centro principale grazie alla sua posizione topografica a nord del fiume Kouris ed ai piedi dei monti dei Troodos, quindi vicino l'area delle miniere, presentandosi come un centro strutturato a cui facevano capo per approvvigionarsi di metalli, ma allo stesso tempo per fornire i propri prodotti agricoli, gli altri insediamenti minori posti nella fascia mediana del fiume Kouris (EpiskopiPhaneromeni, Episkopi-Bambolula, Erimi-Pitharka e Kafkalla, Laonin tou Porakou, solo per il TC I, Kandou, Sotira Linika), che avevano una funzione di seconda lavorazione del metallo, di produzione agricola e stoccaggio, o centri-santuari, villaggi agricoli. Sembra probabile che ci fosse un flusso di merci verso i siti centrali, ma anche un'interazione tra centri secondari e terziari che contribuivano ad uno sviluppo territoriale integrato e gerarchizzato.

Per quanto riguarda il sistema relativo alle valli dei fiumi Caryilis e Germasoia possiamo ipotizzare un sistema simile a quello del Kouris, ma con alcune differenze: non abbiamo un unico centro strutturato come quello di Alassa vicino le miniere ma diverse aree estrattive poco strutturate, con molti centri nella fascia mediana, come attestato dalle diverse tombe sparse nel territorio, e porti/attracchi sulla costa.

Il sistema Pyrgos-Moni, nonostante presenti le stesse caratteristiche morfologiche dei precedenti, ha uno sviluppo insediativo che potremmo definire a clessidra; abbiamo diverse attestazioni ai piedi dei Troodos come nelle aree di Mazokambos ed Asgata per l'estrazione del minerale ma anche un sito, Sanidha, legato alla produzione di ceramica White Slip, legato anche all'estrazione del rame; unitamente ad alcuni siti posti nella media valle del Pyrgos o del Moni, per la produzione e siti-santuario (Kafkalloudi, Katsamouri, Gropuppaes, Laonin tou Nikola $e$ Platias) e siti che rivestono un ruolo sul mare come Agios Georgios che si presenta a tutti gli effetti come un approdo per la navigazione di cabotaggio.

Tale dislocazione funzionale degli insediamenti determinò e condizionò fortemente ciò che avvenne con il passaggio tra il tarda età del bronzo e il primo ferro, quando si verificò una cristallizzazione della segmentazione politico-economica, in cui alcuni siti emersero su altri, sia in aree più costiere che lontani dalla costa anche quelli interni, dando vita a piccoli centri amministrativi con un ruolo funzionale rispetto alle rispettive periferie. Il rame ed i suoi processi estrattivi, di prima lavorazione e poi di lavorazione finale, determinarono la formazione di reti di rapporti e di scambio che a loro volta hanno portato alla creazione dei primi sistemi politici territoriali, ognuno dei quali aveva un centro primario, i quali all'inizio del primo ferro vennero urbanizzati attraverso edifici amministrativi e con l'acquisizione di ceramica d'importazione.

All'inizio del primo Ferro siamo di fronte ad un'epoca segnata da fallimenti urbani ed industriali con una crisi interna e con l'avanzare del fenomeno della migrazione. I siti con complessi "più industriali e monumentali" vengono abbandonati e non più utilizzati (basti pensare ai siti vicini di Kalavassos-Agios Dimitrios e Maroni-Vounes che non furono più riabitati quando la crisi finì), Alassa fu abbandonata e la sua scomparsa colpì la valle del Kouris; vicino la costa sud, Episkopi-Bambolula che funzionava come porto di Alassa per l'esportazione, fu abbandonata nel TC IIIA e non più riabitata. Probabilmente intere regioni, che avevano sviluppato una propria produzione indipendente e scambio nel XIII a.C., non sono riuscite a sopravvivere alla crisi, portando la popolazione alla ricerca di nuove fonti di approvvigionamento delle risorse minerarie. Per quanto riguarda il distretto di Limassol la decadenza di alcuni centri potrebbe esser dovuta al fatto che le vene di 
ferro fossero in altre aree, ad esempio Alassa decade appunto perché non ha ferro e quindi ci si sposta su aree più ricche di tale nuova risorsa mineraria.

Siamo di fronte a centri "vecchi" che lasciano il posto a centri "nuovi" che si affermano grazie ad un diverso livello di specializzazione nei ruoli sul territorio, legati però alla gestione dell'estrazione del ferro e ad una economia agricola probabilmente di tipo più intensivo.

Amathus diviene il sito egemone degli "eteo-ciprioti" in questa lettura di trasformazione di un territorio che non subisce la diaspora, ma che semplicemente si trasforma che non subisce una crisi ma un cambiamento dal punto di vista dell'economia e del commercio in cui il ferro prevale sul rame.

Una trasformazione del quadro insediativo, che, nel distretto di Limassol determinò con il tempo, anche una moltiplicazione delle aree estrattive, senza però che esse si trasformassero in veri e propri centri o villaggi, ma divenendo aree di estrazione direttamente gestite da siti egemoni come Amathus per tutto il distretto orientale di Limasol e Kourion per l'area della Kouris Valley e per tutto il distretto occidentale di Limassol.

\section{Bibliografia}

Catling H.W. 1962, Patterns of settlements in Bronze Age Cyprus. Opuscola Atheniensia 4, pp.129-69.

KNAPP A.B. 1986, Copper production and Divine Protection: Archaeology, Ideology and Social Complexity on Bronze Age Cyprus, SIMA Pochetbook 42, Goteborg P. Astroms Forlag.

Menozzi O. ET AL. 2014, Moni Valley (Cyprus): Survey, Archaeology and Landscape Archaeology, Seasons 2011-2013, in Archaeologia Cypria VII, forthcoming.

ToDD I. 1991, Excavation at Sanidha in RDAC, pp. 75-112.

TODD I. - PILIDES D. 1992, Excavations at Sanidha in RDAC pp. 97-146

TodD I. - PILIDES D. 2000, The Site of Sanidha-Moutti tou Ayiou Serkou in VVP 9: the field survey of the Vasilikos Valley vol.I in Studies of Mediterranean Archeology pp. 161-170.

\section{Contatti autore:}

Dott.ssa Serena Torello Di Nino

Università G.d'Annunzio di Chieti

Mail: serenatorellodinino@gmail.com 
Frankfurter elektronische Rundschau zur Altertumskunde 29 (2016)

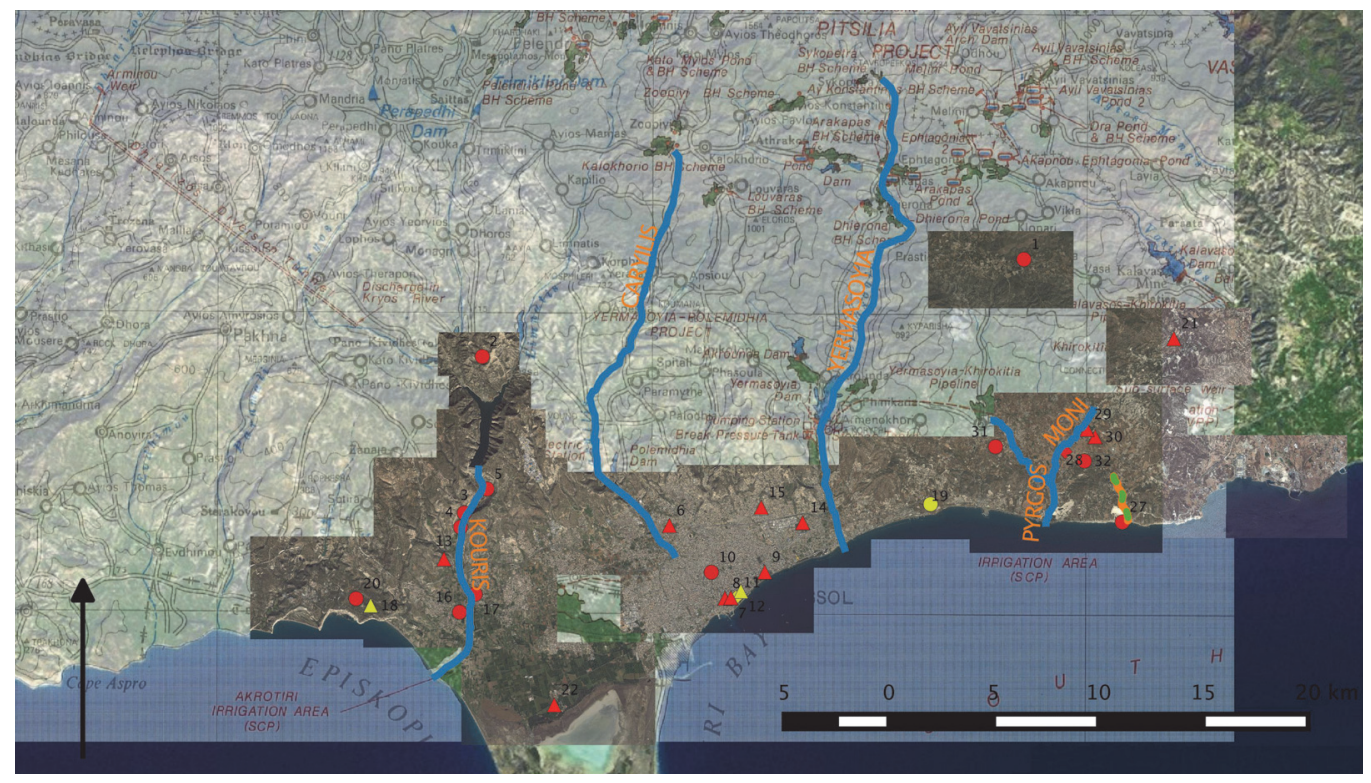

Fig. 1. Immagine del distretto di Limassol realizzata su piattaforma Qgis.

AREA III

LA VALLE DEL PYRGOS-MONI

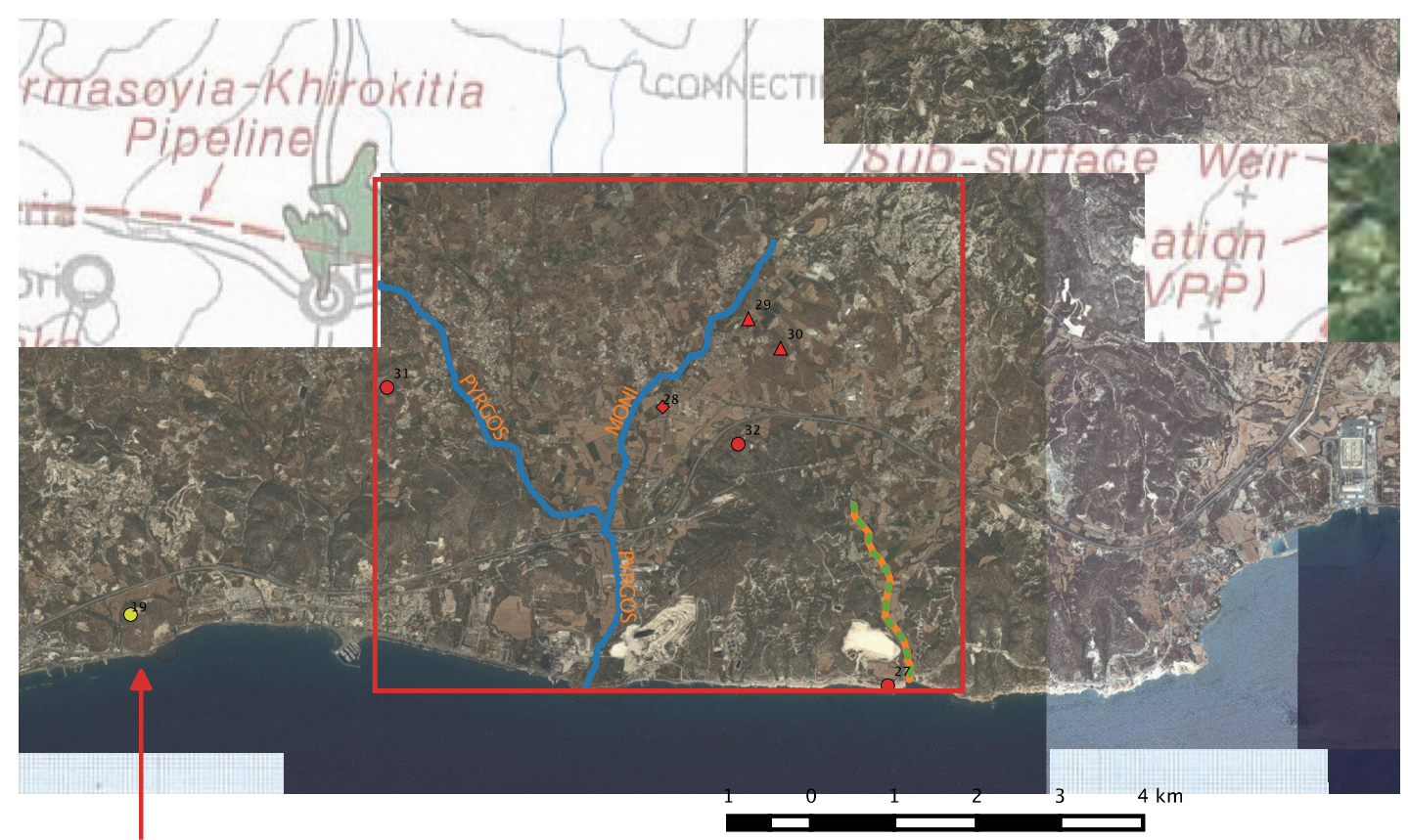

Fig.2. La valle del Pyrgos-Moni. 
Frankfurter elektronische Rundschau zur Altertumskunde 29 (2016)

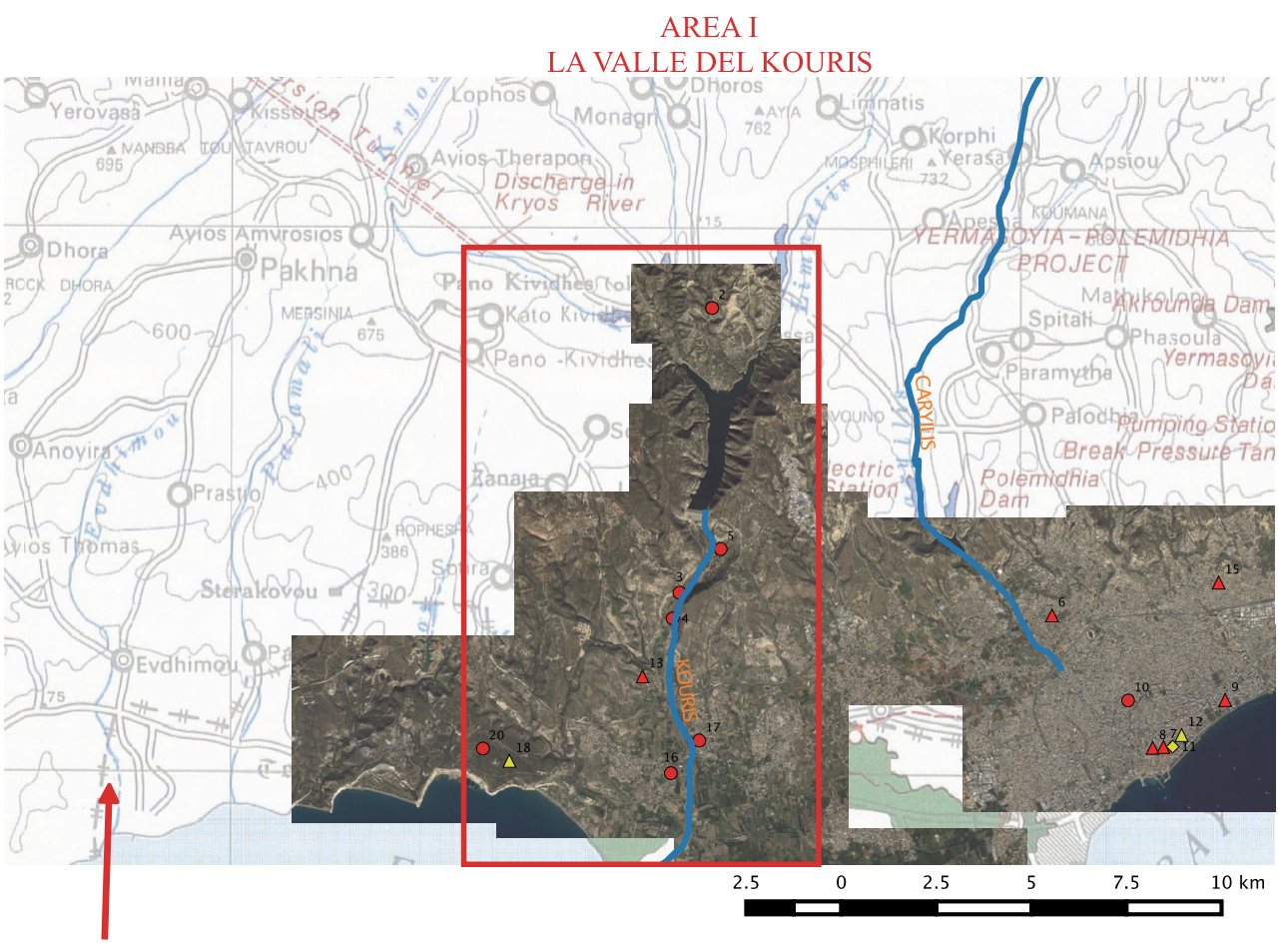

Fig.3. La valle del Kouris. 